\title{
Coerced group collaborative evolution as an explanation for sexual reproduction's prevalence
}

\author{
Robert J. Lin ${ }^{1}$, Feng Lin $^{2^{*}}$ \\ ${ }^{1}$ School of Medicine, Wayne State University, Detroit, USA \\ ${ }^{2}$ College of Engineering, Wayne State University, Detroit, USA; *Corresponding author: flin@ece.eng.wayne.edu
}

Received 10 July 2010; revised 15 August 2010; accepted 18 August 2010.

\begin{abstract}
The prevalence of sexual reproduction has long been an outstanding problem of evolutionary biology. Different explanations have been offered to explain the prevalence of sexual reproduction. These explanations mainly focus on the benefits of sexual reproduction's ability to shuffle and recombine genes. In this paper, we propose an alternative and comprehensive point of view to this important problem. We first hypothesize that sexual reproduction leads to genetic homogeneity and maintains adaptational advantages of organisms. In stable conditions with strong selective pressures, the maintenance of desired adaptational advantages is one benefit of sexual reproduction. We further hypothesize that sexual reproduction provides a mechanism by which entire populations of similar genomes can interact and collaborate with one another in order to improve the population's average genomic fitness, a phenomena we call coerced collaborative group evolution. We show that groups of individuals will improve as a whole, even though each individual is still operating under their own best interests. We also argue that the so-called 'two-fold cost of males' is misguided if we take limited resources in any environment into consideration. Finally, we propose an intuitive and visualized view to connect different theories on sexual reproduction to establish a comprehensive theory to explain sexual reproduction's prevalence.
\end{abstract}

Keywords: Evolution; Sexual Reproduction; Genetic Homogeneity; Selective Pressure; Computer Simulation

\section{INTRODUCTION}

Why sexual reproduction? This question is of interest not only to biologists, but also to the general public as a whole [1-4]. The prevalence of sexual reproduction suggests that there are major benefits provided by this mode of reproduction. The benefits, however, are not obvious. In many ways, asexual reproduction seems to be a better evolutionary strategy: only one parent is required, and all of the parent's genes are passed on to its progeny [5-12]. In a sexual population, the males are unable to produce offspring of their own and females only transfer half their genes to offspring, hence the theoretical problem of the 'two-fold cost of males.' Sexual reproduction must also go through obstacles that do not hinder asexual reproduction. Sexually reproducing organisms must spend a great deal of time and energy to find and attract mates. The peacock is a good example. The male must grow a large and intricate tail to attract mates; not only is producing the tail energy consuming, the peacock must also carry around its tail at all times, leaving it vulnerable to predators. Furthermore, copulation in sexually reproducing organisms leaves both organisms vulnerable to predation. Despite these major drawbacks to sexual reproduction, it is still a very prevalent form of reproduction in most living organisms.

Many researchers have put forth numerous explanations for why sex is so prevalent. Current hypotheses to explain the maintenance of sex typically focus on the benefits of the inherent ability of sexual reproduction to recombine and shuffle genetic information [13-20]. These benefits are undoubtedly significant. We believe, however, there are other explanations for sexual reproduction's prevalence.

We will start by considering various reproductive barriers that must be overcome by sexual reproduction. The consequence is that only parents with similar genetic codes can produce viable offspring, which results in the formation of clusters of individuals with a similar genetic makeup. Thus, sexual reproduction leads to genetic homogeneity of a population. This allows organisms to maintain adaptational advantages. In stable conditions with strong selective pressures, the maintenance of de- 
sired adaptational advantages is one benefit of sexual reproduction [22].

We then show that since in sexual reproduction, each individual will try to find the best mates, groups of individuals will improve as a whole much faster than asexual reproduction, even though each individual is still operating under their own best interests. In this sense, sexual reproduction provides a mechanism by which entire populations of similar genomes can interact and collaborate with another in order to improve the population's average genomic fitness, a phenomena we call coerced collaborative group evolution.

We also argue that since resources in any environment are limited, the assumption that asexual reproduction should have an automatic two-fold reproductive advantage over sexual reproduction is misguided.

Finally, we propose an intuitive and visualized view to connect different theories - including our theory of coerced collaborative group evolution - on sexual reproduction to establish a comprehensive 'poly-theory' to explain sexual reproduction's prevalence.

\section{MAINTENANCE OF ADAPTATIONAL ADVANTAGES BY SEXUAL REPRODUCTION}

One of sexual reproduction's most evident benefits is its ability to allow the recombination of different genes. However, this benefit can only occur when a successful mating takes place. Consequently, intrinsic to the sexual process are numerous reproductive barriers that limit the extent of dissimilarity that can be exchanged between the genetic information of two individuals. These reproductive barriers have been well documented. For a detailed discussion on these reproductive barriers and isolating mechanisms such as ecological isolation, behavior isolation, temporal and mechanical isolation, as well as the prevention of fusion between different species' gametes, see our paper [22].

All these reproductive isolating mechanisms are ultimately derived from an organism's genetic coding. As a result, sexual reproduction indeed allows for genetic exchange, but on the other hand, this genetic exchange cannot be so drastic or profound, otherwise mating will never occur in the first place, or even if it does, the resulting offspring will not survive. Because gene exchange is limited to organisms having compatible genetic codes, we believe that sexual reproduction leads to the formation of clusters of individuals with a similar genetic makeup.

On the other hand, although asexual reproduction is believed to preserve genetic integrity from one generation to the next, the asexual genome is not static and it is prone to various changes such as mutation, horizontal gene transfer, and chromosomal rearrangement [23]. These resulting genetic changes are copied directly to offspring. Any genome change that does not result in an asexual organism's death will be carried into future generations. As a consequence, asexual genomes will diverge and differentiate from each other over time.

Evidence that sexual reproduction promotes genetic homogeneity and that asexual reproduction promotes genetic diversity is not just reserved to the literature. We have created computer simulations that simulate sexual and asexual reproduction, and the results of the simulations provide strong, testable evidence that further supports our hypothesis [22]. As seen from our simulations, asexual reproduction results in diversification of genetic composition and sexual simulations resulted in the formation of tight clusters. These tight clusters are maintained by the sexual process and prevent the massive diversification (deleterious mutation accumulation) seen by asexual simulations.

Now, what is the benefit of genetic homogeneity and clustering? We hypothesize that when conditions are relatively stable, the strength of selective pressures is strong, and when organisms have become adapted to those selective pressures, it would be most beneficial to maintain those adaptations.

To illustrate this benefit of genetic homogeneity and maintenance of adaptational advantages in a biological context, we take the example of a developed ecosystem that is near its "climax community." Such systems can be considered relatively stable with high biodiversity. The high biodiversity means there is more competition for the same limited resources. In order for all these species to successfully live amongst each other, selective pressures have caused each species to develop specific adaptations that allow it to occupy an exclusive niche (strong selective pressure) [24]. It's also a common observation that $\mathrm{k}$-reproductive strategies typically dominate such systems [24]. The practice of organisms in such systems to reproduce fewer, but more highly niche-adapted offspring are all adaptations due to selective pressures that allow these species to survive in this type of environment. Deviating away from such adaptations, which means deviating away from the species' niche, results in competition with other species that occupy other niches. These other species are highly adapted for their niches; consequently, the 'deviant organism' is unlikely to survive the competition. Sexual reproduction maintains adaptational advantages and minimizes the conversion of precious resources to the production of 'deviant organisms' that are unlikely to survive.

The same reasoning applies to an opposite situation. It is a common observation that in harsh environments, 
many sexual organisms employ the r-reproductive strategy. In such environments, specific adaptations geared toward maximizing individual survival in the environment are not as useful. Instead, selective pressures have caused these organisms to develop adaptations that allow them to reproduce quickly and in prodigious numbers, as well as typically having low nutrient requirements, short maturation time, etc. [24]. In order to maintain these adaptations, such organisms also reproduce sexually.

To further support our idea that sex promotes genetic homogeneity and maintains adaptational advantages, we have designed computer simulations that show that sexual reproduction is beneficial in stable conditions with strong selective pressures [22].

In summary, many observations and our computer simulations support the following hypothesis. (1) Sexual reproduction is a species stabilization mechanism that naturally maintains genetic homogeneity and species identity. (2) Asexual reproduction, which does not have this inherent species stabilization mechanism, actually leads to genetic diversity and no definitive species identity. (3) Sexual reproduction is beneficial because the maintenance of species identity maintains desired adaptational advantages, which is important when selective pressures are strong and stable.

\section{COERCED GROUP COLLABORATIVE EVOLUTION BY SEXUAL REPRODUCTION}

We further hypothesize that sexual reproduction is a mechanism by which entire populations of similar genomes can interact and collaborate with another in order to improve the population's average genomic fitness. This process results in descendent offspring adapting much faster and having a higher average fitness than an equivalent asexual population with only natural selection to serve as its adaptive force. In a sense, obligate sexual reproduction acts as the bridging mechanism that causes groups of organisms to evolve together as a group.

We believe that the primary benefit for the maintenance of sexual reproduction is its ability to allow individual genomes within a population, which we shall consequently refer to as a gene pool, to interact and collaborate with one another leading to the gene pool to evolve as a whole unit rather than being the simple sum of all individual genomes comprising it. Naturally, this interaction of genomes comprising the gene pool evolves in such a way that the gene pool adapts to the conditions of its environment. Before we begin, some readers may immediately begin to question our idea due to our mention and emphasis of group evolution. We acknowledge and are aware of the general consensus that group selection is believed to be a relatively minor evolutionary force [25]. Our use of the term group evolution is distinct from group evolution as it applies to group selection. Group evolution, in our sense, is the resulting adaptation of an entire gene pool to its environment through selection forces operating at the level of the individual organism.

Before we begin to explain this, we would like to mention that a hypothesis to explain sexual reproduction must account for the evolution of obligately sexual organisms that don't have the ability to be facultatively sexual as their needs dictate. One can make the argument that the evolution of sexual reproduction naturally leads to the development of obligate sexual reproduction, and that the development of sexual reproduction must incur the complete jettison of all asexual reproductive capacities. However, the presence of such organisms as aphids, etc. clearly shows that the development of sexual reproduction need not necessarily preclude asexual capacities and that the two functions are not necessarily mutually exclusive within the same organism. That clearly begs the question of why sex is not only so prevalent in higher-order organisms, but also why sex tends to be in the obligate form. Why not develop both functions and get the best of both worlds? We shall keep this question in mind, as we develop our concept of coerced collaborative group evolution (CCGE).

We shall now assume a population of hypothetical sexually reproducing organisms. This hypothetical population is genetically diverse enough such that each individual has its own distinctive fitness value in relation to the environment and other individuals within the population. We assume that this population is obligately sexual. We also assume that the primary goal of the individual is to pass on as many of its genes to offspring that will be able to survive to adulthood to spread their own genes.

We shall now focus our attention on a single individual, which we shall refer to as $\mathrm{X}$, within this population. Since this individual is obligately sexual, it automatically incurs the $50 \%$ genetic cost of sexual reproduction. Therefore, in order to maximally spread the $50 \%$ of its genes that it does contribute to offspring, it is in its best interest to mate and reproduce with the most fit individuals in the population that are within reproductive age, all these individuals we shall term generation P. By coupling its genes with a fit individual, $\mathrm{Y}$, in generation $\mathrm{P}$, the resulting offspring, generation F1, will have the best chance of survival to spread their own genes (which are composed of $50 \%$ of X's genetic content and $50 \%$ of Y's genetic content).

Let us consider a single offspring of $\mathrm{X}$, whom we shall refer to as Z. Again, the same principles driving X's mating decisions will also apply to $\mathrm{Z} ; \mathrm{Z}$ will attempt to spread its genes as much as possible in the form of re- 
productively successful offspring. Since $Z$ does not have the option of asexual reproduction, $\mathrm{Z}$ is forced to combine its genes with another individual in generation F1. In order to have the best chances of spreading its genes (which is the combination of $X$ and $Y$ ), it is in the best interests of $\mathrm{Z}$ to mate with the most fit individuals of generation $\mathrm{F} 1$.

We can extrapolate these observations and come to the conclusion that in each generation, the fittest individuals will contribute the greatest proportion to the gene pool, while the least fit individuals would contribute a much lower proportion. Any individual's reproductive success then becomes directly related to that individual's fitness in relation to the average fitness of the gene pool. As a consequence, with each successive generation, the gene pool will shift in content to greater average fitness. This rate of adaptation of the gene pool in accordance to the environment would be faster than if natural selection were to operate alone. This would also lead to greater genetic homogeneity of the population as the fittest individuals leave a disproportionate amount of their genes into the gene pool, much more so than natural selection would achieve by itself.

In sexual reproduction, surviving to reproductive age does not necessarily guarantee an individual's ability to produce offspring; sexual reproduction forces an individual to also be of high fitness (in relation to other individuals in the population) in order to spread its genes. Such a constraint does not apply to asexual reproduction. So long as an asexual individual survives to reproductive age, and so long as the asexual individual has the resources for reproduction, an asexual individual can reproduce at will. In the interests of preserving one's own genes as much as possible, asexual reproduction is clearly the more attractive option: the individual can reproduce at will, and the individual can transfer $100 \%$ of their genes with each offspring. Remove the ability of asexual reproduction, and force all individuals to operate under the same rules (forced mating and 50\% gene transference per offspring) and the result is that the group of individuals operating under those rules will improve as a whole, even though each individual is still operating under their own best interests.

We can immediately see that the simple principles of coerced collaborative group evolution as we have just lain out depend on one simple factor: sexual reproduction must be obligatory. Once an organism acquires the ability to reproduce both sexually and asexually; the overriding desire to spread its own genes as much as possible would most likely cause the organism to opt for asexual reproduction the majority of the time (as this would allow for $100 \%$ gene transference). This would completely erode sexual reproduction's ability to force coerced collaborative group evolution. It is here that we now consider the question of why most sexual organisms are not facultatively sexual? According to our hypothesis, it is because coerced collaborative group evolution cannot operate unless all individuals are obligately sexual. Interestingly, it has also been shown that sexual selection may be a strong enough force to warrant dominance of obligate sexual reproduction over facultative sexual reproduction, even in the short-term [26].

Addressing the issue of sexual reproduction's prevalence requires us to not only provide an explanation of its advantages over asexual reproduction, but also to address the nuances of its mechanics. At its most basic level, sexual reproduction is a process in which an organism halves its genetic content into gametes and then later fuses two gametes to form a new individual. We can easily imagine that the obstacles to overcome in order to develop such a reproductive mechanism are not terribly difficult at the single-cell level. We can suppose that a mutation occurs in a bacterium such that it accidentally splits its genome in half; recovery of its full genomic content would require simple fusing of the daughter cells containing half the genetic content. This is one possible mechanism of the evolution of sex at the unicellular level. Undoubtedly, there are other possible ways to develop such a mechanism. That however, is not our main concern.

What concerns us is that this concept simply illustrates that at the most basic level, sex simply requires a halving of genes into gametes, and then a fusion of these gametes to form a full genome. The simple mechanics of this process applies to the ever-increasing complexities of higher-level organisms. At the most basic level of this process, designation of a male or female component is arbitrary and irrelevant. From this basic concept, one can easily imagine evolution progressing in such a manner that the male/female differentiation process remains irrelevant; that eventually, hypothetical animals in this hypothetical divergent evolution could evolve in such a manner as to produce both eggs and sperm. Copulation could occur such that both animals release sperm that fertilize with eggs in both respective individuals. In such a process, differentiation of gametes into eggs and sperm may not even have evolved in the way that we understand eggs and sperm to be; however, that is speculation and not central to this thought process. Supposing such a reproductive mechanism did evolve and both organisms using this mechanism would both be impregnated and bare the burden of producing children. A population of these hypothetical organisms would no longer have half the population (the males) being unable to produce children. Such a population would have the equivalent fecundity as an asexual population; the only limitation being the time and energy to recruit a suitable mate. Such a process would bypass the supposed 'two-fold 
cost of males,' while retaining all the recombination benefits that sexual reproduction provides. We hope the reader will agree that such a dual-copulation process of sexual reproduction, aka monoeciousism in animals, is very possible in theory. In fact, earthworms are a natural example of animals that engage in the type of monoecious sexual reproduction that we have just described.

With this hypothetical thought experiment that we have just lain forth, we conclude that not only must there be a reasoning behind the development of sexual reproduction in higher level organisms, but there must also be a reasoning for why animals have predominantly developed a dioecious rather than a monoecious form of sexual reproduction. A strong explanation for sex should logically be able to explain both questions in one hypothesis.

Our hypothesis of coerced collaborative group evolution, in which obligate sexual reproduction forces all organisms in a population to behave through individual interests in such a manner that causes the group gene pool to move towards maximal average fitness much faster and more efficiently than natural selection alone, is able to answer both of those questions. We have already explained how the mechanisms of coerced collaborative group evolution lead to greater gene pool fitness. Therefore, we now seek to answer why animals have predominantly chosen a dioecious form of sexual reproduction rather than the monoecious form that many plants have adopted.

According to our hypothesis, mate selection plays an important role in the mechanics governing coerced collaborative group evolution. Each individual organism seeks to maximize the spread of its genes, and this is best achieved if that individual's genes are coupled with the genes of a high fitness individual. A central difference between animals and plants is the issue of mobility. Animals, with their ability to move around and actively sift through potential mates within their geographic location are much better equipped to successfully identify and mate with high fitness individuals of the same species. Plants, which don't have that same mobility, must rely on far less discriminating means of spreading their gametes: insect pollination, etc. By losing the ability of mate selectivity, the advantages provided by coerced collaborative group evolution are reduced; hence providing an incentive to choose monoecious sexual reproduction over dioecious sexual reproduction.

One can certainly make the argument that more fit plants are more likely to attract insect interaction, through more lustrous displays of flowers, and hence in theory would spread their genes to more plants. However, with no ability of receiving flowers to distinguish between the quality of pollen being deposited, the power of coerced collaborative group evolution in plants is almost certainly reduced in comparison to animals. The question of how much coerced collaborative group evolution is at work in plants is admittedly difficult for us to address with certainty. However, we will simply point out that plant species have developed to be both dioecious and monoecious, while animals are almost certainly dioecious. The fact that plants can be both dioecious and monoecious does not work against our hypothesis, while the fact that animals are almost certainly dioecious lends support to our hypothesis.

We have previously lain forth a hypothetical form of monoeciousism in animals of 'dual-copulation' in which one copulation event results in both individuals becoming impregnated by the other individual. We chose to start with that example because of its simplicity; some readers may point out that there are other possible hypothetical forms of monoeciousism in animals. An obvious example would be an organism that has both male and female reproductive organs and one copulation event between two individuals resulting in only one individual becoming impregnated, rather than a dual-copulation event. Once again, like the dual-copulation example, this would be a reproductive strategy in which all members of the population could contribute to the production of offspring; effectively bypassing the 'two-fold cost of males.' Once again, we must ask ourselves, why did the evolution of animals not proceed down this evolutionary pathway? And once again, an explanation for the maintenance of sexual reproduction must be able to resolve this question.

Just like the assumptions we made for the foundational mechanisms of coerced collaborative group evolution, let us assume that each individual acts in a selfish manner to maximize the spread of their genes. Once again, individuals are obligately sexual and always contribute $50 \%$ of their genes for every offspring produced. In this scenario, unlike dual-copulation, each individual has two choices: (1) be 'male,' and simply provide DNA for fertilization and bear no further reproductive costs or (2) be 'female,' and provide DNA and bear the reproductive costs of producing the offspring (and possibly expending further resources to raise the offspring to adulthood).

It should be clear that if every individual is to act in a manner that maximizes spread of their genes, then option 1 would be the logical choice that every individual would choose; the amount of genes provided per copulation is equivalent, and without having to expend further resources raising the offspring, the individual is free to expend those resources in further copulation events. However, if every individual selected option 1 as their strategy, then there would be no individuals producing 
offspring. Clearly, such a scenario is not viable and this may be sufficient to explain why this form of monoecious sexual reproduction is not prevalent.

For the sake of argument, let us assume that not every individual chooses option 1 , thereby allowing the production of offspring and the continued survival of the species. Now we must ask, what would cause an organism to choose option 2 over the more attractive option 1 ?

One possibility is a 'domination' scenario. Before a copulation event, both individuals engage in some sort of battle, with the victor gaining the right to engage in option 1. In such a contest, it would be logical to assume that the stronger or more fit individual would win the contest and thereby force the weaker, less fit individual, to bear the reproductive burden of option 2 . If every individual is out to maximize the spread of their genes, then we think it's logical that every individual of every level of fitness would seek to mate with an individual of lower fitness. This reasoning is simple, by mating with a lower fitness individual, the organism increases its chances of mating by option 1 without paying the costs of option 2. However, this strategy runs exactly opposite of our proposed coerced collaborative group evolution, and such cumulative actions of seeking lower fitness individuals would lead to a degradation of the average fitness of the gene pool over time. We are unaware of any animals that engage in this type of monoecious sexual reproduction, giving us some confidence in the validity of coerced collaborative group evolution.

Another possibility is a 'random sexual function' scenario. In this case, the choosing of the 'male' between two individuals is chosen at random. Since the individuals have no choice over the matter of who is placed with the burden of option 2, all individuals will naturally choose to mate with individuals of high fitness. Such a strategy would promote similar positive effects of coerced collaborative group evolution on the gene pool. However, such a scenario is unlikely in nature because it is not an evolutionarily stable strategy. Mutations allowing individuals to choose the male sex would be selected for, bringing the species back into the problems that we have already described.

A third possibility is a 'desperation' scenario. In every copulation event, the individual that chooses option 2 does so voluntarily. However, we can assume that each individual will first attempt option 1 as their default strategy. Individuals will refrain from doing option 2 unless they decide that option 2 is their best strategy because producing some offspring is better than producing no offspring. What would cause an individual to decide that option 2 is a preferable strategy than option 1 ? That would depend on the individual's history of reproductive success with option 1 in relation to the time left in the individual's fertile phase of their life cycle. We assume that every individual will voluntarily choose to do option 2 at the very end of their fertile phase; the individual might as well engage in one last-ditch effort to produce one more batch of offspring. This means that in a population of varying aged individuals, there will always be some individuals willing to choose option 2 , all other individuals will choose to do option 1 and will be competing amongst each other for mating rights. Each individual that volunteers for option 2 will naturally want to mate with the highest fitness individual in order to maximize offspring survival. This means that the highest fitness individuals will have the highest success with option 1. Individuals with high success with option 1 will naturally continue to choose the strategy of option 1. Individuals that have minimal success with option 1 , individuals of low fitness, are more likely to decide that option 2 is a preferable reproductive strategy earlier in their fertile phase, and then will likely continue with maintaining option 2 as their strategy. This leads to an interesting conclusion: high fitness individuals will almost always be coupled to low fitness individuals in every copulation event. If that's the case, then the average fitness of the gene pool will improve very slowly, if at all, compared to a population engaged in coerced collaborative group evolution with designated sexes. That no organisms engage in this type of reproduction, gives us more confidence in coerced collaborative group evolution.

As with the case of nearly all universally accepted biological principles, the incredible diversity and complexity of life always lends to a few exceptions; hermaphroditism in animals is no exception. We will attempt to explain these unusual forms of sexual reproduction within the framework of coerced collaborative group evolution.

Our first case of interest is the simultaneous hermaphrodites, in particular, earthworms that we have previously mentioned, that engage exactly in the dual-copulation mechanism that we have described. At this point, we can only offer a weak explanation similar to the explanation of monoecious plants. Earthworms are motile and hence have one advantage over plants in a possible utilization of mate selection to result in a coerced collaborative group evolution process; however, their sensory systems (necessary for mate selection) are much more limited in capacity than dioecious animals. Their sensory systems are limited to tactile sensation, limited sensitivity to light, and chemical receptors. With limited ability for mate selection, there is no impetus to drive their evolution towards utilization of coerced collaborative group evolution. Some may point out that there are a whole host of dioecious organisms with sen- 
sory systems more limited than the earthworm. However, these organisms are also capable of asexual reproduction in one form or another, and hence we've omitted them from discussion. Therefore, like the example of monoecious plants, whether or not a species adopts dioecious sexual reproduction is an interplay between the strength of selection for coerced collaborative group evolution due to its positive modulating effect on the species' gene pool and the higher reproductive capability of monoecious sexual reproduction.

We will now focus our attention on the sequential hermaphodites and the systems of protandry and protogyny. A good example of protandry is the clownfish in which each fish is originally born male. These fish generally live in a group in which there is one large reproductive female, a smaller reproductive male, and many smaller non-reproductive males. Upon death or removal of the female, the reproductive male changes into the female, and the largest non-reproductive male becomes reproductive male. Such interesting reproductive behaviors actually work to enhance the effect of coerced collaborative group evolution. The numbers of females are dramatically reduced compared to the normal 1:1 Fisherian sex ratio. Meanwhile, it is the largest (fittest) non-reproductive male that becomes the reproductive male upon conversion of the reproductive male to female. This phenomena is essentially an enhancement of coerced collaborative group evolution, in which only the two most highly fit individuals of the group contribute their genes to all consequent offspring of the group. Why most organisms have not chosen this reproductive mode is most likely due to the high social interaction required of the species that participates in this reproductive form, as well as the reduced reproductive fecundity of having such small numbers of offspring-bearing females. This example offers evidence in support of coerced collaborative group evolution as well as brings into question the validity of the 'two-fold cost of males,' as we will discuss later in this paper.

An example of the process of protogyny is the blue wrasse. These fish are born either males or females in Fisherian ratios. However, there are two types of males: terminal phase males and initial phase males. Initial phase males are non-reproductive and look very similar to females. Death or removal of the terminal phase male results in either the dominant female or the dominant initial phase male becoming the terminal phase male. Such a process actually leads to an enhancement of coerced collaborative group evolution, in which highly fit females also possess the ability to become the reproductive terminal phase male and consequently can spread their genes to a much greater extent in benefit for the gene pool. However, the probability that the fittest fe- male has a better fitness than the fittest male of the group is probably very small, and so the added enhancement of coerced collaborative group evolution is probably minimal at best. The greater simplicity of purely dioecious sexual reproduction for a minimal loss of coerced collaborative group evolution effect probably explains the predominance of pure dioecious sexual reproduction over this form of protogyny.

\section{ISSUE OF TWO-FOLD COST OF MALES}

We would now like to focus discussion on the 'two-fold cost of males.' The principle of the 'two-fold cost of males' is based essentially on the assumption that because a dioecious sexually reproducing population is half male with no offspring bearing capabilities, then an asexually reproducing population in which all members can bear offspring should be able to generate much more offspring and hence outcompete a sexually reproducing population; therefore, sexual reproduction must have a two-fold fitness advantage over asexual reproduction. At first glance, it would seem that this assumption appears to be logically sound. It is on this assumption that numerous theories have been offered that attempt to illustrate how sexual reproduction can potentially lead to a two-fold fitness advantage of its offspring.

However, it is of our opinion that the assumption that asexual reproduction should have an automatic two-fold reproductive advantage over sexual reproduction is misguided. The problem is that a sexually reproducing species can always double its production of offspring, thereby equaling the overall reproductive rates of an asexual population. If sexual reproduction can always reach a reproductive rate equal to asexual reproduction, then the numerous advantages conferred by sex that have been elaborated by numerous scientists as well as coerced collaborative group evolution that we have just described, are more than enough to warrant sexual reproduction's continued maintenance and prevalence.

Obviously, one can make the immediate argument that for every doubling of reproductive rates of a sexual population, an asexual population could also double its rate to outcompete the sexual population. This would be true for a scenario in which an environment has infinite resources, and this is why the initial argument for the 'two-fold cost of males' appears at first glance to be sound. However, as we know, any environment has a limited amount of resources; an upper limit has to be reached, otherwise, resources would be so diluted that no organism would be able to survive. Therefore, one can imagine that in the reproductive 
rate arms race between an asexual and a sexual population, an equilibrium point where the reproductive rate of the sexual population will be about the same as an asexual population will eventually be achieved. One can imagine that at such an equilibrium point, an asexual population, being at a fitness disadvantage against a sexual population, may try to make that up by increasing its reproductive rate. However, to do so would decrease its survival and reproductive success in the immediate future (as well as the sexual population) due to the limiting factor of finite resources, thereby driving its reproductive rate back to equilibrium; the same can be said for a sexual population increasing its reproductive rate in order to match any asexual attempt at an increased reproductive rate advantage.

Now, these are all hypothetical arguments on paper, and we believe that natural phenomena are the best evidence. Going back to our previous arguments of monoecious and dioecious sexual reproduction, we pointed out that a monoecious form of sexual reproduction would bypass the reproductive rate disadvantage of dioecious sexual reproduction. So why is dioecious sexual reproduction still common? If we are to hypothetically assume that our coerced collaborative group evolution arguments are not true, then one is once again left with the question of why dioecious sexual reproduction is more prominent over monoecious sexual reproduction. Based on the observation of so much dioecious sexual reproduction, one can argue that the cost of males must not be very large; otherwise natural selection would have surely driven evolution towards the direction of monoecious sexual reproduction.

\section{TOWARD A COMPREHENSIVE THEORY TO EXPLAIN SEXUAL REPRODUCTION'S PREVALENCE}

In the quest to answer the mystery of sex, many competing theories have been offered. No doubt, some proponents of each of these respective theories may justifiably cling to their belief that their theory is the best explanation among this group of theories. However, none of the current theories seem truly satisfactory when viewed in isolation. Also, none of these theories are truly mutually exclusive of each other. As such, we are of the belief that a true explanation for sexual reproduction's prevalence will likely require a 'poly-theory' synthesis of existing explanations for sex.

We will attempt to unify our theory of coerced collaborative group evolution within this 'poly-theory' framework. Before doing so, we would like to emphasize that while this new framework may seem to be an attempt on our part to push our theory as 'the grand the- ory of all things sex,' that is not the case at all. We have already emphasized that our theory of coerced collaborative group evolution attempts to address the specific question of obligate dioecious sexual reproduction and we have already acknowledged that in cases of monoecious sexual reproduction or facultative sexual reproduction, coerced collaborative group evolution plays a lesser role. In those cases, we believe a 'poly-theory' explanation of sex is needed to explain sexual reproduction's multiple advantages over asexual reproduction.

To explain coerced collaborative group evolution and how it fits among the other theories of sex, we will address each theory on an individual basis, and show how the theory plays a role in coerced collaborative group evolution and gene pool group evolution. In order to aid our discussion, we would like to first put forth a model to help visualize each theory and how it fits with coerced collaborative group evolution. Let us imagine a two dimensional space (we choose two dimensions for simplicity). Within this space we can place a point at any arbitrary location. This point can then be viewed as representing the $100 \%$ maximal fitness genotype location, with the surrounding space representing all possible genotypic points. Of course, this $100 \%$ maximal fitness genotype point is completely hypothetical; we simply make the assumption that if all biotic and abiotic factors in a complex ecosystem can be accounted for and quantified, then there should be one hypothetical genotype that would be perfectly adapted for those parameters, this is the $100 \%$ maximal genotype point. Any point located in the surrounding space is deviating from perfect fitness, with points being closer to the maximal point being higher in fitness than points at a location with a farther radius. We can then think of a 'gene pool' being the sum of all these points. Average fitness of the gene pool would be measured by summing all of the radial point distances from the maximum fitness point and then averaging the sum; the lower the average, the higher the average fitness of the gene pool.

Natural selection can be thought of as an 'inward' force that pushes genotypic points closer to the maximum fitness point. Genotype points closer to the maximum fitness have a higher chance of producing offspring (generating genotype points within the vicinity of the parent genotype point); genotype points farther away have a lower chance of producing offspring, and hence, over time the location of genotype points will shift closer to the maximum point. The results of this model and natural selection's effect on how the model works should be very underwhelming to the reader; we are simply taking a time-cherished theory and restating it under a different perspective; some readers may already 
visualize natural selection in a manner similar to how we've just described it.

Mutation can be thought of as a primarily 'outward' force that pushes genotypic points away from the maximum fitness point. Neutral mutations would simply result in the generation of a genotypic point within the same circumferential radius as the parent point. Deleterious mutations results in points generated at a farther radius. Beneficial mutations result in points with a closer radius. However, it's generally agreed that most mutations are neutral or deleterious hence, we view mutation as an 'outward' force.

With this model, we must also factor in that environments are never static; biotic and abiotic factors are always in a constant state of flux. Under our model, this can be represented by our maximum fitness point being under a constant state of motion. Under a short time scale, this point would make minimal movements around a general vicinity of the genotypic space. However, under a long time scale, or the introduction of a catastrophic event, the movement of the maximum fitness point from its original location can be significant.

With this model in mind, our first focus will be on the most basic theory of sex: promotion of genetic variation. The random contribution of $50 \%$ of the genes of both parents in sexual reproduction leads to the generation of varying genotypes amongst the offspring. This allows greater exploration of genotype possibilities hence providing the raw fuel for natural selection to act upon. In addition, these varying genotype possibilities interact with the environment to contribute to varying phenotypes, upon which mating selectivity can act upon, thereby fueling the process of coerced collaborative group evolution. This generation of greater phenotypic substrate variety that can be acted upon is the foundation for the mechanics of natural selection and coerced collaborative group evolution. In essence, under our visual model, this promotion of genetic variation allows parents to generate offspring at different genotypic points than themselves. These offspring points can be generated at radial distances closer, farther, or the same as the parent points in relation to the maximum fitness point.

Our next focus will be on the concept of Muller's Ratchet. Under this theory, continued accumulation of deleterious mutations leads to a degradation of a species' average fitness over time, without any method of correction, the species will likely face extinction [27]. Muller's Ratchet explains that sex allows good genes in separate loci to be recombined to restore a genotype to optimal fitness. Under our model, accumulation of deleterious mutations is represented by genotypic points gradually shifting away from the maximum fitness point. The recombination of good genes back onto a single genotype would be represented by two outwardly shifted genotype points creating an offspring that has a genotype point shifted inwards. This shifted genotype then has the possibility of reproducing with other genotypic points to help shift the average fitness of the gene pool back towards the maximum fitness point. Coerced collaborative group evolution dictates that since this point has a higher fitness than other points, the genotypic content of this point is more likely to be reproductively successful, meaning more offspring points are going to be generated at a location closer to the maximum fitness point. Overall, this results in greater genotypic point density closer to the maximum fitness point. Hence, coerced collaborative group evolution helps to enhance improvement of the gene pool average fitness.

Another theory is Kondrashov's Hatchet, or the deterministic mutation hypothesis. In this theory, recombination of deleterious genes onto a single individual results in a synergistic fatal effect for that individual. These individuals will have much more reduced fitness and more unlikely to survive to reproductive age; as a result, their deleterious genes are more likely to be reduced from the population [28]. Coerced collaborative group evolution would posit that even if these individuals survived to reproductive age, their reduced fitness would give them a much smaller chance of reproductive success, thereby enhancing the probability that their deleterious genes will be removed from the gene pool. In essence, these individuals face two filters to reproductive success: natural selection and mate selection. In simplified terms within the context of our model, Kondrashov's Hatchet would function as an 'inward' force that counters the outward force of mutation, thereby increasing the genotypic point density closer to the maximum fitness point (increasing average fitness).

Another widely touted theory is the Red Queen Hypothesis. In this theory, parasites and hosts engage in a constant arms battle with hosts evolving resistances to parasites and parasites evolving ways to get past those resistances $[10,29]$. In this theory, sex then generates new genotypes at a much faster rate than would be possible with asexual reproduction. The faster generation of new genotypes then allows for rapid adaptation of resistances against parasites for the hosts. Likewise, sexually reproducing parasites can have rapid adaptation against host resistances. Under our model, this constant adaptation and counter-adaptation can be viewed as having the maximum fitness point shifting its point within the genotypic space at a rapid pace. A rapidly shifting maximum fitness point means that the group gene pool must constant shift its area in the genotypic space in response to movements of the maximum fitness point. Under the perspective of coerced collaborative group 
evolution, mate selectivity results in faster group gene pool modulation in response to the rapidly shifting maximum fitness point location.

In Section 2, we hypothesized that sexual reproduction is a species stabilization mechanism that naturally maintains genetic homogeneity and species identity. This is beneficial because the maintenance of species identity maintains desired adaptational advantages, which is important when selective pressures are strong and stable. In a sense, sexual reproduction functions as a filter that prevents mutational deviants from contributing their genetic content back into the group gene pool. In effect, this reduces the outward force of mutations (genetic homogeneity) within the context of our model. The numerous well-studied genetic anomalies in humans and the resulting sterility that often accompany these conditions are an excellent example of deleterious mutations being barred from entering the group gene pool. In cases of genetic abnormalities of organisms that remain fertile and also manage to evade the filter of natural selection, these organisms are unlikely to be successful in sexual reproduction. This also leads to coerced collaborative group evolution.

\section{CONCLUSIONS}

In this paper, we explained sexual reproduction's prevalence by making the following arguments. 1) Sexual reproduction maintains adaptational advantages of organisms. 2) Sexual reproduction provides a mechanism for coerced collaborative group evolution. 3) The so called 'two-fold cost of males' is misguided. 4) The best way to explain sexual reproduction's prevalence is by a comprehensive 'poly-theory'.

\section{ACKNOWLEDGEMENTS}

This research is supported in part by the NSF under grants ECS-0624828 and ECS-0823865, and by the NIH under grant 1R01DA022730. We would like to thank Hao Ying for his continued support and encouragement of our idea and Robert D. Brandt for his advice and guidance.

\section{REFERENCES}

[1] Bell, G. (1982) The Masterpiece of Nature: The Evolution and Genetics of Sexuality. University of California Press, USA.

[2] Colegrave, N. (2002) Sex releases the speed limit on evolution. Nature, 420, 664-666.

[3] Hurst, L.D. and Peck, J.R. (1996) Recent advances in understanding of the evolution and maintenance of sex. TREE, 11, 46-52.

[4] Misevic, D., Ofria, C. and Lenski, R.E. (2006) Sexual reproduction reshapes the genetic architecture of digital organisms. Proceedings of Royal Society B, 273, 457-
464.

[5] Agrawal, A.F. (2006) Evolution of sex: Why do organisms shuffle their genotypes. Current Biology, 16, R696-R704.

[6] Bachtrog, D. (2006) A dynamic view of sex chromosome evolution. Current Opinion in Genetics and Development, 16, 578-585.

[7] Chasnov, J.R. (2000) Mutation-selection balance, dominance and the maintenance of sex. Genetics, 156, 1419-1425.

[8] de Visser, J.A.G.M. and Elena, S.F. (2007) The evolution of sex: Empirical insights into the roles of epistasis and drift. Nature Reviews Genetics, 8, 139-149.

[9] Dolgin, E.S. and Otto, S.P. (2003) Segregation and the evolution of sex under overdominant selection. Genetics, 164, 1119-1128.

[10] Ladle, R.J. (1992) Parasites and sex: catching the red queen. Trends in Ecology Evolution, 7, 405-408.

[11] Otto, S.P. (2003) The advantages of segregation and the evolution of sex. Genetics, 164, 1099-1118.

[12] Otto, S.P. and Lenormand, T. (2002) Resolving the paradox of sex and recombination. Nature Reviews Genetics, 3, 252-261.

[13] Agrawal, A.F. (2001) Sexual selection and the maintenance of sexual reproduction. Nature, 411, 692-695.

[14] Agrawal, A.F. and Chasnov, J.R. (2001) Recessive mutations and the maintenance of sex in structured populations. Genetics, 158, 913-917.

[15] Arkhipova, I. and Meselson, M. (2004) Deleterious transposable elements and the extinction of asexuals. BioEssays, 27, 76-85.

[16] Barton, N.H. and Charlesworth, B. (1998) Why sex and recombination? Science, 281, 1986-1990.

[17] Charlesworth, B. (1993) Directional selection and the evolution of sex and recombination. Geneticd Research, 61, 205-224.

[18] Elena, S.F. and Lenski, R.E. (1997) Test of synergistic interactions among deleterious mutations in bacteria. Nature, 390, 395-398.

[19] Feldman, M.W., Christiansen, F.B., and Brooks, L.D. (1980) Evolution of recombination in a constant environment. PNAS, 77, 4838-4841.

[20] Kondrashov, A.S. (1988) Deleterious mutations and the evolution of sexual reproduction. Nature, 336, 435-440.

[21] Lin, R. (2005) A new hypothesis on the evolutionary advantage of sexual reproduction. Proceedings of the 8th Joint Conference on Information Sciences (JCIS 2005). Salt Lake City, Utah, USA, 21-26 July 2005, 18-21.

[22] Lin, R. and Lin, F. (2008) Simulation study to explain sexual reproduction's prevalence. International Journal of Simulation: Systems, Science and Technology, 9, 4352.

[23] Alberts, B., Bray, D., Hopkin, K., et al. (2004) Essential cell biology. 2nd Edition, Garland Science, New York.

[24] Raven, P.H., Johnson, G.B., Losos, J.B., et al. (2005) Biology. 7th Edition, McGraw-Hill, New York.

[25] Maynard Smith, J. (1964) Group selection and kin selection. Nature, 201, 1145-1147

[26] Hadany, L. and Beker, T. (2007) Sexual selection and the evolution of obligatory sex. BMC Evolutionary Biology, $7,245$. 
[27] Muller, H.J. (1964) The relation of recombination to mutational advantage, Mutat. Res., 1, 2-9.

[28] Elena, S.F., Carrasco, P., et al. (2005) Mechanisms of genetic robustness in RNA viruses. European Molecular Biology Organization 7, 168-173.

[29] Hamilton, W.D., et al. (1990) Sexual reproduction as an adaptation to resist parasites. PNAS 87, 3566-3573

[30] Kondrashov A.S. (1994) Muller's ratchet under epistatic selection. Genetics 136, 1469-1473.
[31] Heng, H.H. (2007) Elimination of altered karyotypes by sexual reproduction preserves species identity. NRC Canada Genome 50, 517-524.

[32] Ridley M. (1997) Evolution. Oxford University Press.

[33] Zeyl, C. and Bell, G. (1997) The advantage of sex in evolving yeast populations. Nature 388, 465-468.

[34] Hoekstra, R.F. (2005) Why sex is good. Nature 434, 571-573. 